\title{
Dynamic Characteristics Improvement of Digital Controlled DC-DC Converter by Means of PZC Control
}

Seiya Abe ${ }^{1}$, Mariko Ogawa ${ }^{1}$, Toshiyuki Zaitsu ${ }^{2}$, Satoshi Obata ${ }^{2}$, Masahito Shoyama ${ }^{1}$, Tamotsu Ninomiya

1 Kyushu University

2 Texas Instruments Japan Ltd.

3 Nagasaki University

E-mail: abe@ees.kyushu-u.ac.jp

\begin{abstract}
Recently, the performance of the DSP and FPGA is developed remarkably. So, fully digital control is enabled in switch mode power supplies. However, in many cases, the control system is built by very complicatedly and very difficult theories such as the adaptive control, predictive control, and so on. Furthermore, in most popular PID control, its design method of the parameters is not clear, so derivation of the optimal parameters is very difficult. This paper proposes revolutionary control technique which is cancelled the transfer function of the converter completely by using pole-zero-cancellation method. This technique is very simple and easy to stability design.
\end{abstract}

\section{INTRODUCTION}

In recent years many electronic circuits is digitized. However, only switch mode power supplies are consisted of the analog circuit until now. This is because the switch mode power supplied consisting of analog circuit is realized with low cost. However, almost circuits are digitized and the whole system is operated by processors. Therefore, it is difficult for switch mode power supplies to stay in the system as the analog circuit. Hence the digitization of the switch mode power supplies is necessary.

So far, various examinations have been discussed about digital control of switch mode power supplies[1-5]. However, important parameters such as the switching frequency were impractical because the performance of processors such as DSP or FPGA was not so good.

Recently, due to the development of the semiconductor manufacture technology, the performance of processors is developed remarkably. Therefore, expectation of the realization of the fully digital control switching power supply becomes higher.

However, the control system is built by very complicatedly and very difficult theories such as the adaptive control or predictive control in many cases of the discussions of the digital controlled switch mode power supplies so far. On the other hand, also in the most popular and easiest control method such as PID control, the design method is not so clear, and the optimal design is difficult $[6,7]$.
This paper proposes revolutionary control technique which is cancelled the transfer function of the converter by using polezero-cancellation method. This technique is a method based on classical control theory and digital redesign. Moreover, this technique is very simple and easy to stability design of converter system. Furthermore, the arbitrary frequency characteristics can be created by introducing a new frequency characteristic. Here, the design method and system stability of the proposed control technique is examined by using buck converter.

\section{DYNAMIC CHARACTERISTICS ANALYSIS}

For the design of the control system, it is necessary to grasp correctly the characteristics of the converter in detail. The buck converter as the controlled objects is shown in Fig. 1. The dynamic characteristics of each converter can be derived by applying the state space averaging method[8,9]. The dynamic characteristic of duty to output voltage of each converter is derived following equation;

$$
G_{d v}(s)=\frac{\Delta V_{o}(s)}{\Delta D(s)}=\frac{G_{d v o}(s)}{P(s)}
$$

where;

$$
\begin{aligned}
& P(s)=\frac{s^{2}}{\omega_{o}^{2}}+s \frac{2 \delta}{\omega_{o}}+1 \\
& G_{d v o}(s)=\left(\frac{s}{\omega_{e s r}}+1\right) \frac{R}{R+r_{L}} V_{i}
\end{aligned}
$$

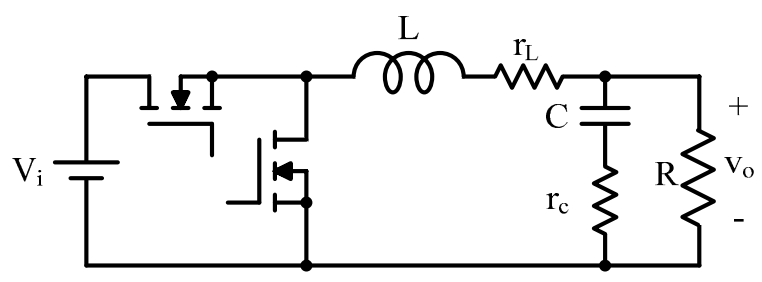

Fig. 1. Buck converter. 
$\omega_{o}=\sqrt{\frac{R+r_{L}}{L C\left(R+r_{c}\right)}}$

$\delta=\frac{L+C\left\{R r_{c}+r_{L}\left(R+r_{c}\right)\right\}}{2 \sqrt{L C\left(R+r_{c}\right)\left(R+r_{L}\right)}}$

$\omega_{\text {esr }}=\frac{1}{C r_{c}}$

Figure 3 (a) shows the construction of the analog system, and Fig. 3 (b) shows the block diagram of analog system. From, Fig. 3, the loop gain of analog controlled converter can be derived following equation;

$$
T(s)=\frac{\Delta V_{o}(s)}{\Delta V_{o}^{*}(s)}=\frac{G_{d v o}(s)}{P(s)} \cdot G_{c}(s) \cdot K \cdot K_{s} \cdot P W M
$$

where;

$\mathrm{Gc}(\mathrm{s})$ : Transfer function of phase compensator

$\mathrm{K}: \mathrm{DC}$ gain of error amp.

Ks : Sense gain of output voltage

PWM : transfer gain of voltage to duty

In order to evaluate the accuracy of analytical results, the analytical results are compared with experimental results. Table. 1 shows the circuit parameters and specifications. Figure 4 shows the frequency response of analog controlled buck converter with p-control. As shown in Fig. 4, both results agreed well. However, in digital control case, the phase characteristic at high frequency side is different greatly as shown in Fig. 5.

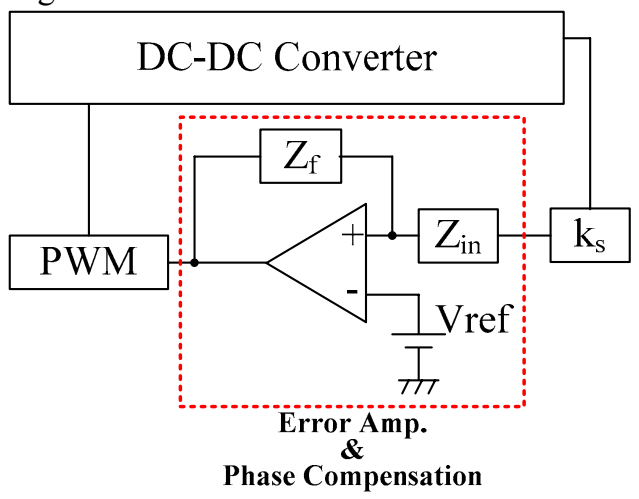

(a) Construction of analog control system.

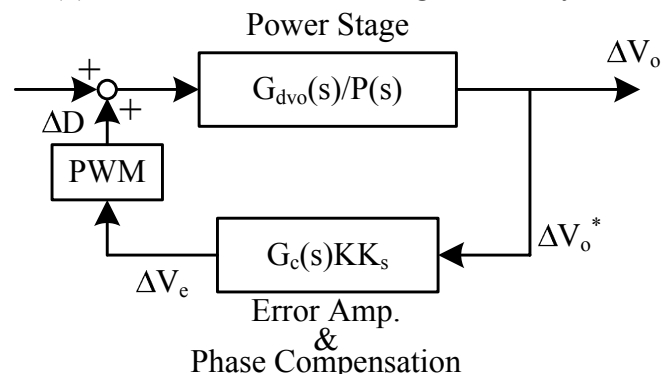

(b) Block diagram of analog controlled system

Fig. 3. Analog control system.
TABLE I

Circuit parameters and specifications

\begin{tabular}{|c|c|c|}
\hline Symbol & Description & Value \\
\hline $\mathrm{Vi}$ & Input Voltage & $12 \mathrm{~V}$ \\
\hline $\mathrm{Vo} / \mathrm{Io}$ & Load Condition & $2.5 \mathrm{~V} / 5 \mathrm{~A}$ \\
\hline $\mathrm{L}$ & Filter Inductor & $22 \mu \mathrm{H}$ \\
\hline $\mathrm{C}$ & Filter Capacitor & $470 \mu \mathrm{F}$ \\
\hline $\mathrm{r}_{\mathrm{L}}$ & DC Resistance of L & $100 \mathrm{~m} \Omega$ \\
\hline $\mathrm{r}_{\mathrm{c}}$ & ESR of C & $25 \mathrm{~m} \Omega$ \\
\hline $\mathrm{R}$ & Load Resistance & $1 \Omega$ \\
\hline $\mathrm{K}_{\mathrm{s}}$ & Sense Gain & 0.25 \\
\hline $\mathrm{K}$ & Feedback DC Gain & 5 \\
\hline PWM & PWM Gain & 0.5 \\
\hline $\mathrm{f}_{\mathrm{s}}$ & Switching Frequency & $100 \mathrm{kHz}$ \\
\hline $\mathrm{T}_{\text {sample }}$ & Sampling Period & $10 \mu \mathrm{s}$ \\
\hline
\end{tabular}

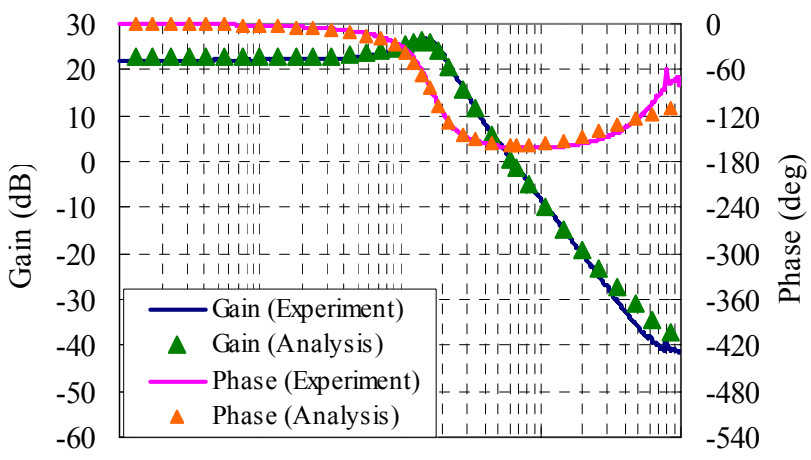

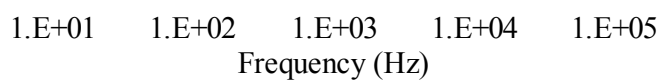

Fig. 4. Frequency response comparison of analytical and experimental results (analog p-control).

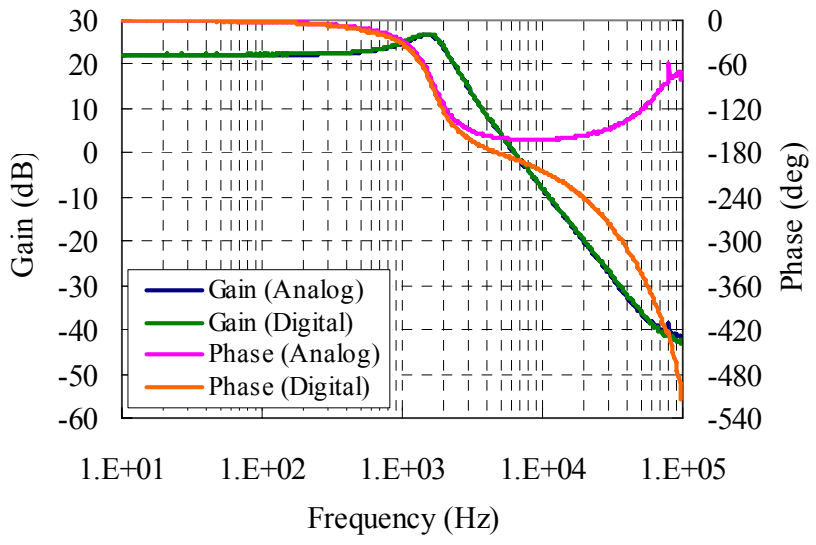

Fig. 5. Frequency response comparison of analog and digital control (Experimental results). 
In digital control system, the output voltage as a detected signal is converted to discrete signal by $\mathrm{AD}$ converter. And then, the detected signal is calculated by DSP. Moreover, the calculated signal decides the duty ratio of next switching period. Therefore, the detected signal is reflected by the next switching period. As mentioned above, the delay of one period is included into the control loop as a dead time element as shown in Fig. 6. From Fig. 6, the loop gain of digital controlled converter can be derived following equation;

$$
T(s)=\frac{\Delta V_{o}(s)}{\Delta V_{o}^{*}(s)}=\frac{G_{d v o}(s)}{P(s)} \cdot G_{c}(s) \cdot H_{e}(s) \cdot K \cdot K_{s} \cdot P W M
$$

where;

$$
H_{e}(s)=e^{-s T_{\text {sample }}}
$$

$\mathrm{Gc}(\mathrm{s})$ : Transfer function of phase compensator

$\mathrm{K}$ : DC gain of error amp.

Ks : Sense gain of output voltage

PWM : transfer gain of voltage to duty

$\mathrm{He}(\mathrm{s})$ : Dead time component of digital controller

Figure 7 shows the frequency response of dead time element $\mathrm{He}(\mathrm{s})$. As shown in Fig. 7, the gain is not changed on frequency response. However, the phase is rotating around 180 degrees at Nyquist frequency $(=\mathrm{fs} / 2)$, and the phase is rotating around 360 degrees at switching frequency (sampling frequency).

In order to evaluate these discussions, the experiment results and analytical results are compared using the specifications and parameters in Table 1. Figure 8 shows the frequency response comparison of analytical and experimental results in digital control case. As shown in Fig. 8, both results agreed well.

In analog control case, the phase rotation of frequency response is improved at higher frequency side by influence of ESR-Zero, and the system has stable operation, as shown in Fig. 4. In this case, the phase margin is around 23 degrees. On the other hand, the digital control case, the phase of frequency response is drastically rotated by influence of the dead time component $\mathrm{He}(\mathrm{s})$. As a result, the phase margin disappears, and the system becomes unstable, as shown in Fig. 6 .

In digital control case, the phase rotation is larger than analog control case by influence of dead time component $\mathrm{He}(\mathrm{s})$, so the phase compensation is necessary to keep the system stability.

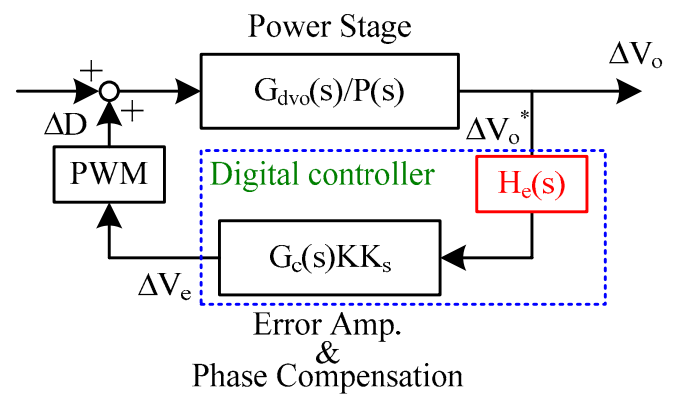

Fig. 6. Block diagram of digital control system including dead time element.

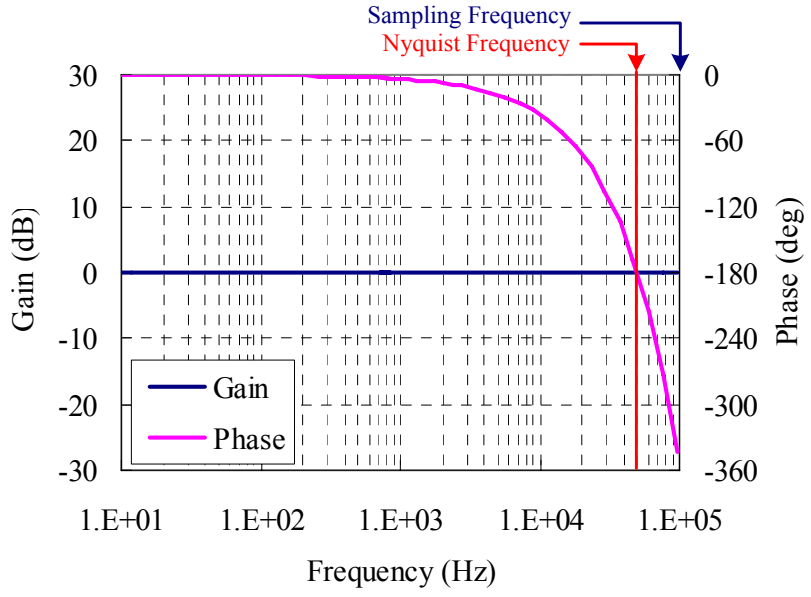

Fig. 7. Frequency response of $\mathrm{He}(\mathrm{s})$ (Analytical result).

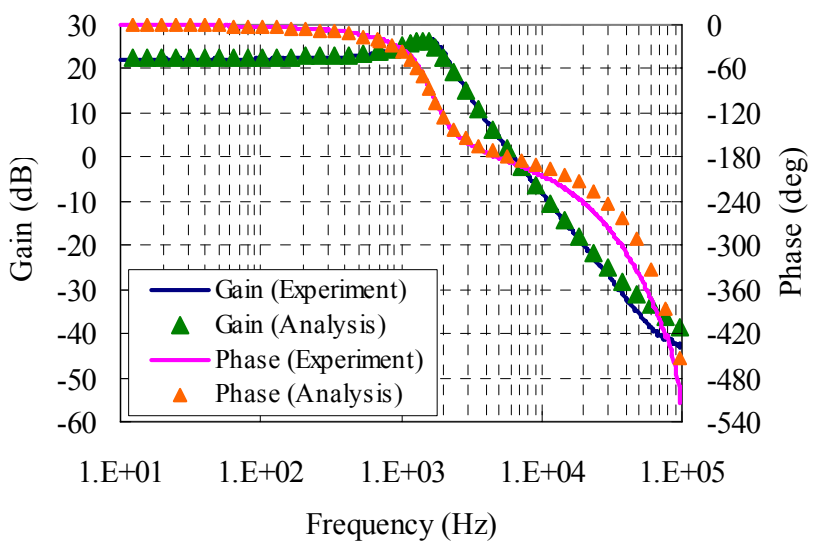

Fig. 8. Frequency response comparison of analytical and experimental results (digital p-control).

\section{Phase COMPENSATION}

The phase compensation is usually used for improvement of the system stability. There are various phase compensation. Above all, the phase lead-lag compensation is the most popular and easiest technique in analog control. Here, the digital filter is build by digital redesign. The phase lead-lag compensation is used as the digital filter. The conversion to digital filter uses bilinear z-transform from analog filter. The transfer function of phase lead-lag compensation is shown in Eq. (9). In order to implement the digital filter into DSP, Eq (9) is converted by using bilinear z-transform as shown in Eq (10).

$$
G_{c}(s)=\frac{\Delta v_{e}}{\Delta v_{o}^{*}}=\frac{\left(\frac{s}{\omega_{z 1}}+1\right)\left(\frac{s}{\omega_{z 2}}+1\right)}{\left(\frac{s}{\omega_{p 1}}+1\right)\left(\frac{s}{\omega_{p 2}}+1\right)}
$$


$G_{c}(z)=\frac{\Delta v_{e}}{\Delta v_{o}{ }^{*}}=k \frac{z^{-2} B_{2}+z^{-1} B_{1}+B_{0}}{z^{-2} A_{2}+z^{-1} A_{1}+A_{0}}$

where;

$$
\begin{aligned}
& k=\frac{\omega_{p 1} \omega_{p 2}}{\omega_{z 1} \omega_{z 2}}(11) \\
& A_{0}=\frac{4}{T_{\text {sample }}^{2}}+\frac{2\left(\omega_{p 1}+\omega_{p 2}\right)}{T_{\text {sample }}}+\omega_{p 1} \omega_{p 2} \\
& A_{1}=-\frac{8}{T_{\text {sample }}^{2}}+2 \omega_{p 1} \omega_{p 2} \quad(13) \\
& A_{2}=\frac{4}{T_{\text {sample }}^{2}}-\frac{2\left(\omega_{p 1}+\omega_{p 2}\right)}{T_{\text {sample }}}+\omega_{p 1} \omega_{p 2} \\
& B_{0}=\frac{4}{T_{\text {sample }}^{2}}+\frac{2\left(\omega_{z 1}+\omega_{z 2}\right)}{T_{\text {sample }}}+\omega_{z 1} \omega_{z 2} \\
& B_{1}=-\frac{8}{T_{\text {sample }}^{2}}+2 \omega_{z 1} \omega_{z 2}(16) \\
& B_{2}=\frac{4}{T_{\text {sample }}^{2}}-\frac{2\left(\omega_{z 1}+\omega_{z 2}\right)}{T_{\text {sample }}}+\omega_{z 1} \omega_{z 2}
\end{aligned}
$$

Figure 9 shows the analytical results of loop gain with phase lead-lag compensation. Where the filter parameters are set as follows; fp $1=10 \mathrm{~Hz}, \mathrm{fz} 1=50 \mathrm{~Hz}, \mathrm{fp} 2=20 \mathrm{kHz}, \mathrm{fz} 2=700 \mathrm{~Hz}$. As shown in Fig. 9. The bandwidth is around $10 \mathrm{kHz}$, and the phase margin is around 40degrees. Moreover, this system has stable operation. Figure 10 shows the experimental results of loop gain with phase lead-lag compensation. In this case, the bandwidth is around $10 \mathrm{kHz}$, and the phase margin is around 30degrees. Both results agreed well.

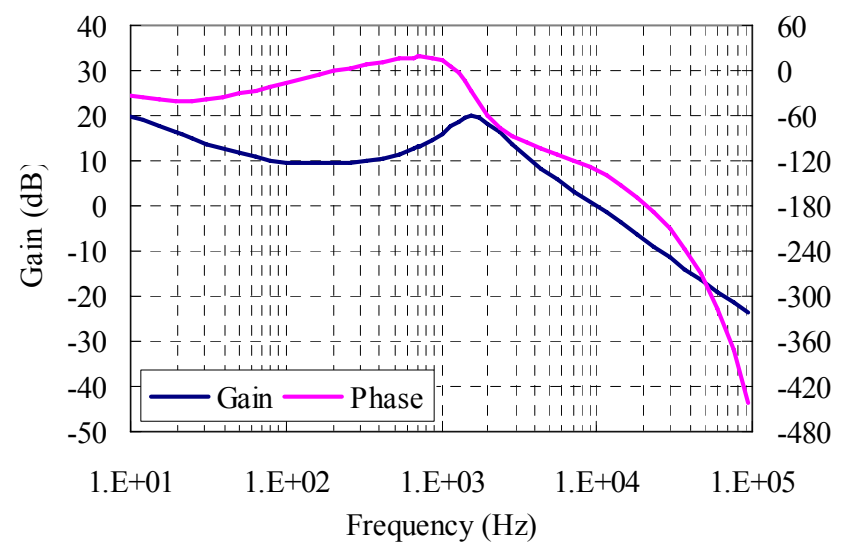

Fig. 9. Analytical results of loop gain with phase lead-lag compensation.
From these discussions, the controller design using classical control theory needs to observe the frequency response of control objects. Moreover, the controller design needs knowledge and experience for system stability. If there is no characteristic of power stage, the controller design becomes very easy.

\section{PZC CONTROL TEChNiQue}

Reduction of the phase rotation is very important for system stability. Especially in the second order system, the phase is drastically rotated around 180 degrees at resonance peak. The stability of the system is improved remarkably if the phase rotation can be reduced.

This paper proposes revolutionary control technique which is cancelled the transfer function of the converter by using polezero-cancellation method. The phase rotation and gain change can be suppressed by canceling the converter characteristics. Furthermore, new characteristic is set in the system by designing the arbitrary transfer function.

Figure 11 shows the block diagram of pole-zero-cancellation control. From Fig. 11, the transfer function of compensator is given following equation;

$$
G_{c}(s)=G_{\text {new }}(s) \cdot G_{p z c}(s)
$$

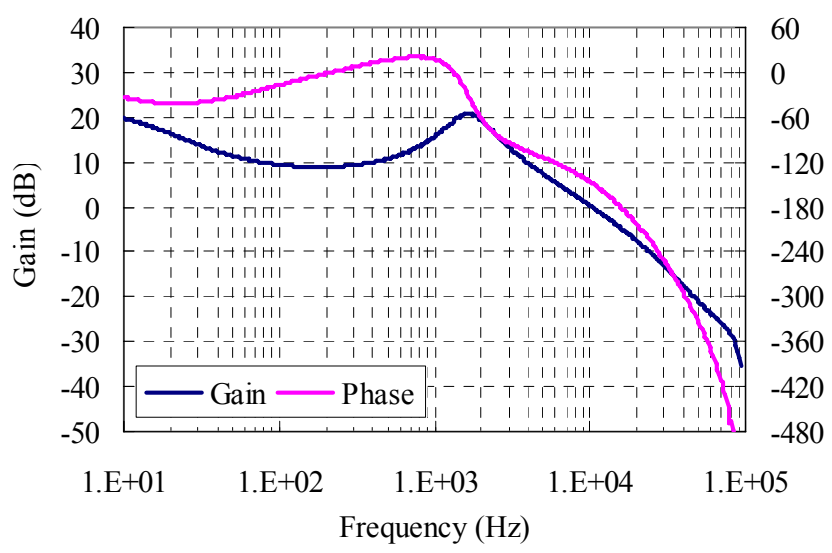

Fig. 10. Experimental results of loop gain with phase lead-lag compensation.

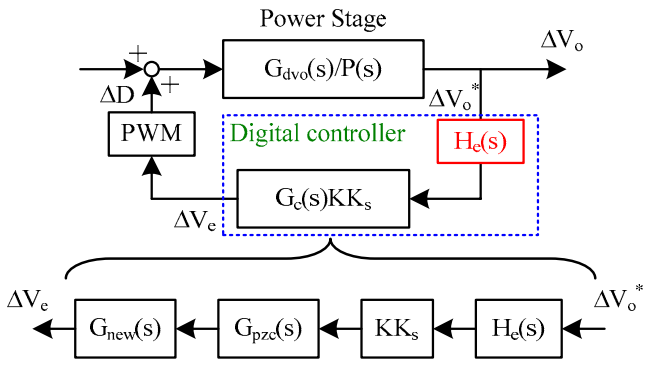

Fig. 11. Block diagram of PZC control. 
Here, the Gnew(s) is defined as simple low pass filter.

$$
G_{\text {new }}(s)=\frac{1}{\frac{s}{\omega_{c}}+1}
$$

In buck converter case, the resonance peak and ESR-Zero are cancelled. The phase rotation of 180 degree is reduced by cancelling resonance peak. The transfer function of the polezero-cancellation $\mathrm{Gpzc}(\mathrm{s})$ is given following equation;

$G_{p z c}(s)=\frac{\frac{s^{2}}{\omega_{o}^{2}}+s \frac{2 \delta}{\omega_{o}}+1}{\frac{s}{\omega_{e s r}}+1}$

Moreover, the transfer function of the compensator is given following equation;

$G_{c}(s)=\frac{\frac{s^{2}}{\omega_{o}^{2}}+s \frac{2 \delta}{\omega_{o}}+1}{\left(\frac{s}{\omega_{e s r}}+1\right)\left(\frac{s}{\omega_{c}}+1\right)}$

In order to implement the digital filter into DSP, Eq (21) is converted by using bilinear z-transform as shown in Eq (22).

$G_{c}(z)=\frac{\Delta v_{e}}{\Delta v_{o}^{*}}=\frac{z^{-2} B_{2}+z^{-1} B_{1}+B_{0}}{z^{-2} A_{2}+z^{-1} A_{1}+A_{0}}$

where;

$$
\begin{aligned}
& A_{0}=\frac{4 / \omega_{\text {esr }} \omega_{c}}{T_{\text {sample }}^{2}}+\frac{2\left(1 / \omega_{\text {esr }}+1 / \omega_{c}\right)}{T_{\text {sample }}}+1 \\
& A_{1}=-\frac{8 / \omega_{\text {esr }} \omega_{c}}{T_{\text {sample }}{ }^{2}}+2(24) \\
& A_{2}=\frac{4 / \omega_{\text {esr }} \omega_{c}}{T_{\text {sample }}^{2}}-\frac{2\left(1 / \omega_{\text {esr }}+1 / \omega_{c}\right)}{T_{\text {sample }}}+1 \\
& B_{0}=\frac{4 / \omega_{o}^{2}}{T_{\text {sample }}^{2}}+\frac{4 \delta / \omega_{o}}{T_{\text {sample }}}+1 \\
& B_{1}=-\frac{8 / \omega_{o}^{2}}{T_{\text {sample }}^{2}}+2 \quad(26) \\
& B_{2}=\frac{4 / \omega_{o}^{2}}{T_{\text {sample }}^{2}}-\frac{4 \delta / \omega_{o}}{T_{\text {sample }}}+1
\end{aligned}
$$

Figure 12 shows the frequency response of the compensator. Where the filter parameters are set as follows; $\mathrm{fc}=1.1 \mathrm{kHz}$. As shown in Fig. 12, the ant resonance peak is appeared at the same frequency of power stage frequency response. Figure 13 show the analytical results of loop gain. As shown in Fig. 13, the bandwidth is around $18 \mathrm{kHz}$, and the phase margin is around 30degrees. Moreover, this system has stable operation. Figure 14 shows the experimental result of loop gain. In this case, the bandwidth is around $17 \mathrm{kHz}$, and the phase margin is around 25 degrees. Both result agreed well. From Fig. 13, 14, the characteristic of the buck converter is canceled and shows only the characteristic of the low pass filter. Moreover, the slope of gain curve is $-6 \mathrm{~dB} /$ oct, so this system is first order system.

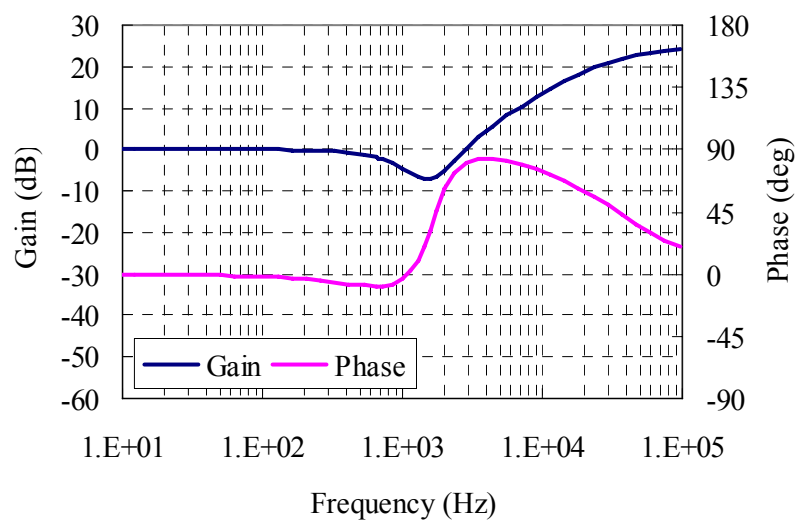

Fig. 12. Frequency response of $\mathrm{Gc}(\mathrm{s})$.

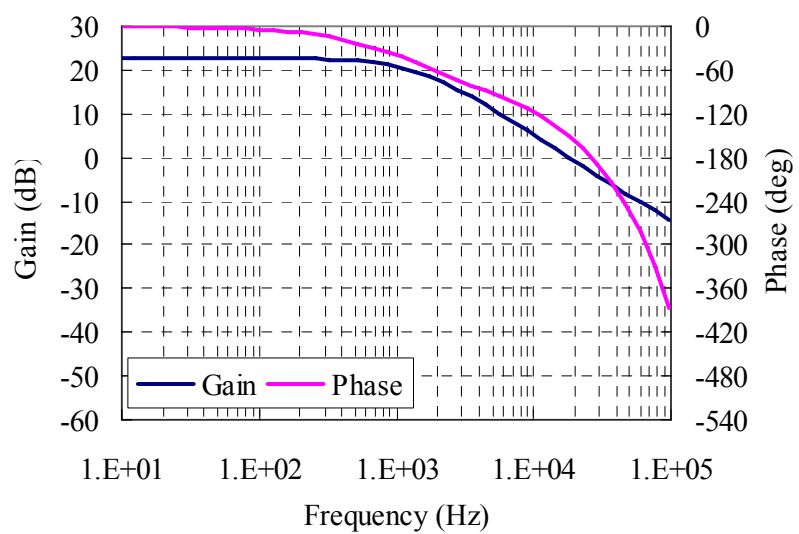

Fig.13.Frequency response of loop gain with PZC control (Analytical results)

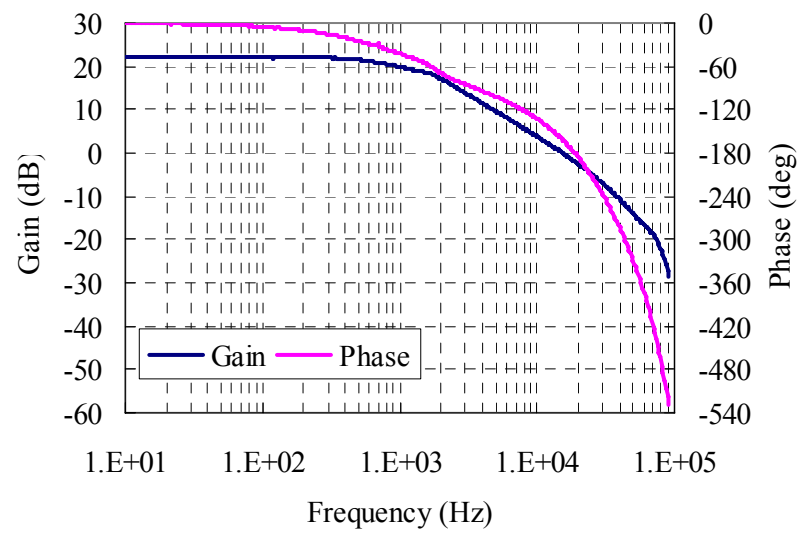

Fig.14. Frequency response of loop gain with PZC control (Experimental results) 


\section{CONCLUSIONS}

This paper proposes revolutionary control technique which is cancelled the transfer function of the converter by using polezero-cancellation method. This technique is very simple and easy to stability design of converter system. Furthermore, the arbitrary frequency characteristics can be created by introducing a new frequency characteristic.

Here, the design method and system stability of the proposed control technique is examined analytically and experimentally by using buck converter. As a result, the effectiveness of proposed control technique is confirmed. Moreover, it is confirmed that the characteristic cancellation of the power stage transfer function can be realized very easy and can be set the arbitrary characteristic.

\section{REFERENCES}

[1] Philip T. Krein, "Digital Control Generations -- Digital Controls for Power Electronics through the Third Generation," IEEE PEDS'07, pp P-1P5, 2007

[2] A. Kelly and K. Rinne, "Control of DC-DC Converters by Direct Pole Placement and Adaptive Feedforward Gain Adjustment," IEEE APEC'05, pp - , 2005.

[3] A. Kelly, K. Rinne,"A Self-Compensating Adaptive Digital Regulator for Switching Converters Based on Linear Prediction," IEEE APEC'06, pp 712-718, 2006.

[4] Y. Wen, S. Xiao, Y. Jin, I. Batarseh, "Adaptive Nonlinear Compensation for Asymmetrical Half Bridge DC-DC Converters," IEEE APEC'06, pp 731-736, 2006.

[5] L. Guo, J. Y. Hung, and R. M. Nelms, "Digital controller design for buck and boost converters using root locus," IEEE IECON'03, pp. 1864-1869, 2003.

[6] H. Guo, Y. Shiroishi, and O. Ichinokura, "Digital PI controller for high frequency switching DC/DC converter based on FPGA," IEEE INTELEC'03, pp-536-541, 2003.

[7] M. He, J. Xu, "Nonlinear PID in Digital Controlled Buck Converters," IEEE APEC'07, pp 1461-1465, 2007.

[8] R.D. Middlebrook, S. Cuk, "A General Unified Approach to Modeling Switching-Converter Power Stages," IEEE Power Electronics Specialists Conference (PESC) 1976, pp. 18-34.

[9] T. Ninomiya, M. Nakahara, T. Higashi, K. Harada, “A Unified Analysis of Resonant Converters," IEEE Transactions on Power Electronics Vol. 6. No. 2. April 1991, pp. 260-270. 